\title{
Magneto-optical properties of charged excitons in quantum dots
}

\author{
C. Schulhauser, ${ }^{1}$ D. Haft, ${ }^{1}$ R. J. Warburton, ${ }^{2}$ K. Karrai ${ }^{1}$ A. O. Govorov, ${ }^{1,3,4}$ A. V. Kalameitsev, ${ }^{4}$ A. Chaplik, ${ }^{4}$ W. Schoenfeld ${ }^{5}$ \\ J. M. Garcia, ${ }^{6}$ and P. M. Petroff ${ }^{5}$ \\ ${ }^{1}$ Center for NanoScience and Sektion Physik, Ludwig-Maximilians-Universität, Geschwister-Scholl-Platz 1, D-80539 München, Germany \\ ${ }^{2}$ Department of Physics, Heriot-Watt University, Edinburgh EH14 4AS, United Kingdom \\ ${ }^{3}$ Department of Physics and Astronomy and CMSS Program, Ohio University, Athens, Ohio 45701 \\ ${ }^{4}$ Institute of Semiconductor Physics, RAS, Siberian Branch, 630090 Novosibirsk, Russia \\ ${ }^{5}$ Materials Department, University of California, Santa Barbara, California 93106 \\ ${ }^{6}$ Instituto de Microelectronica de Madrid, CNM-CSIC Isaac Newton, 8, PTM, 28760 Madrid, Spain \\ (Received 28 May 2002; revised manuscript received 20 August 2002; published 8 November 2002)
}

\begin{abstract}
We present results on the influence of a magnetic field on excitons in semiconductor quantum dots, concentrating on the diamagnetic curvature. We use samples with a bimodal ensemble photoluminescence (PL) and we find that for the low-energy PL branch, the diamagnetic curvature is independent of charge, yet for the high-energy branch, the diamagnetic curvature is strongly reduced with excess charge. Guided by model calculations, we interpret the two classes as typical of the strong and intermediate confinement regimes. In the light of this, we predict that in the weak confinement regime the excitonic diamagnetic shift is strongly dependent on surplus charge, corresponding to a reversal in sign of the conventional diamagnetic shift for neutral excitons.
\end{abstract}

DOI: 10.1103/PhysRevB.66.193303

PACS number(s): 78.67.Hc, 73.40.Rw, 78.66.Fd

A semiconductor quantum dot (QD) represents an ideal model system for the investigation of quantum mechanical electron-electron interactions. This is because Coulomb blockade allows electrons to be added or removed one by one simply with a gate electrode. ${ }^{1,2}$ As a result, the electrical, ${ }^{2}$ optical, ${ }^{3-6}$ and magnetic properties ${ }^{7}$ are tunable. An exciton complex consists of a hole bound to the electrons in a QD. The spatial extent of the excitonic wave function reflects the joint effects of the QD's confinement potential and the Coulomb interactions and can be probed by applying a magnetic field $B$. For neutral excitons, the exciton energy increases quadratically with $B$, the so-called diamagnetic shift, with a curvature proportional to the area of the wave function. ${ }^{8}$ However, the behavior of charged excitons is less well-known and potentially much more interesting because of the more elaborate Coulomb interactions.

Here, we address both experimentally and theoretically the effect of electron charging on the excitonic diamagnetic shift. We show that an additional charge leads to a new paramagnetic contribution. Unlike paramagnetism in solids and atoms, we propose that the QD paramagnetism is a signature of strong Coulomb interactions. The charged exciton we investigate is very easily ionized in both homogeneous bulk semiconductors and quantum wells and so by turning to QD's we have entered a new regime where the Coulomb interactions can dominate the response to a magnetic field.

For the experiments, we used self-assembled InAs QD's, where it is well known that excitons recombine efficiently through photon emission ${ }^{9}$ and that their charge can be controlled electrostatically. ${ }^{1}$ The InAs QD's are grown by molecular beam epitaxy and are embedded $25 \mathrm{~nm}$ above a highly doped GaAs layer, the back contact, and $150 \mathrm{~nm}$ below a Schottky gate on the surface. The QD's are grown in the Stranski-Krastanow mode, giving at our growth temperature $520^{\circ} \mathrm{C}$ lens-shaped QD's. We then deposit $1 \mathrm{~nm}$ of GaAs before annealing the sample at the growth temperature for 1 min. ${ }^{10}$ Ensemble photoluminescence (PL) experiments show that the annealing step produces a bimodal distribution of dots: there are two dominant PL bands, one centered at $1.31 \mathrm{eV}$ (the red band) and one at $1.34 \mathrm{eV}$ (the blue band). A bimodal QD distribution has been observed before, notably in the $\mathrm{Ge} / \mathrm{Si}$ system, ${ }^{11}$ but also for annealed InAs quantum dots, ${ }^{12}$ but we exploit this property as it allows us to study the effects of different confinement strengths within the same sample. The ground state PL of the red-band dots exhibits a small diamagnetic shift, on average $10 \mu \mathrm{eV} / \mathrm{T}^{2}$, and there is an excited state transition some $52 \mathrm{meV}$ above the ground state PL. Both of these facts imply that the excitons are highly localized. Conversely, the PL of the blue-band QD's exhibits diamagnetic shifts on average twice those of the red-band dots, implying that the excitons in the blue-band dots are less localized. The energy difference between the red-band and blue-band dots is caused predominantly by the vertical confinement. We have evidence from Stark shift measurements that the blue-band dots have a slightly softer vertical potential than the red-band dots, ${ }^{13}$ implying that the red-band and blue-band dots have different indium compositions.

We excite the PL by generating carriers in the wetting layer with a $822 \mathrm{~nm}$ laser diode. We measure the PL from single quantum dots by processing $300 \mathrm{~nm}$ diameter apertures in the otherwise opaque metal gate, collecting the PL with a confocal microscope at $4.2 \mathrm{~K}$ and up to $B=9 \mathrm{~T}$. In all the experiments, the pump intensity was low enough that emission from biexcitons was undetectable.

The basic experiment is to measure the PL energy as a function of gate voltage $V_{g}$ and magnetic field. We observe a redshift in the PL whenever a single electron tunnels from the back contact into the QD, and from the Coulomb blockade plateau in the PL we can unambiguously determine the charge of the exciton. ${ }^{3}$ The QDs emitting near $1.26 \mathrm{eV}$ on the red side of the red-band PL acquire one extra electron at 


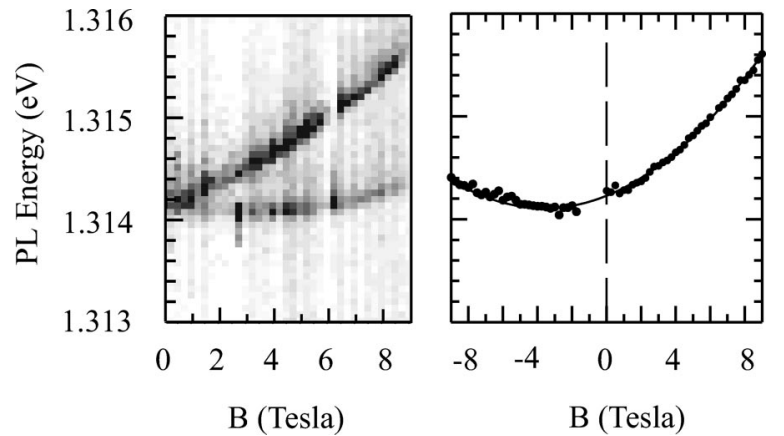

FIG. 1. Left: gray scale plot of the photoluminescence (PL) intensity against magnetic field $B$. Black corresponds to 240 counts in $120 \mathrm{sec}$ on the detector; white is the background signal. Right: the peak positions of the upper and lower branches shown left plotted against positive and negative $B$, respectively. The solid line is a fit of the energy to a second order polynomial in $B$.

$V_{g} \sim-0.65 \mathrm{~V}$ and can be filled with as many as three electrons before the charge spills out into the wetting layer. For the QD's emitting near $1.37 \mathrm{eV}$ on the blue side of the blueband PL, the depth of the confinement potential is substantially less such that the charging threshold moves to $V_{g} \sim$ $-0.15 \mathrm{~V}$, and only one extra electron can be added. In high magnetic field, there is only a small change in the gate voltages at which charging occurs so that we can be sure the charge remains constant as we change the magnetic field.

A typical $B$ dependence of the PL is shown in Fig. 1. The PL line splits into two in magnetic field through the Zeeman effect. The splitting is $120 \mu \mathrm{eV} / \mathrm{T}$, varying by $\pm 30 \mu \mathrm{eV} / \mathrm{T}$ from dot to dot, without any measurable dependence on excitonic charge. In order to analyze the overall up shift of the PL in $B$, we plot the upper (lower) branch against positive (negative) $B$ values. Such a representation reveals the quadratic dependence of the PL peak shift $\Delta E_{\mathrm{PL}}=g_{e x} \mu_{B} B / 2$ $+\alpha B^{2}$, where $g_{e x}$ is the Landé factor and $\mu_{B}$ the Bohr magneton. We focus on $\alpha$ which we have measured for about 20 different QD's.

Figure 2 shows the PL dispersion of two different QD's, one from the red band of the ensemble PL, and one from the blue band, for different excitonic charges. The red-band QD has $\alpha=10 \pm 1 \mu \mathrm{eV} / \mathrm{T}^{2}$, independent of the excitonic charge,
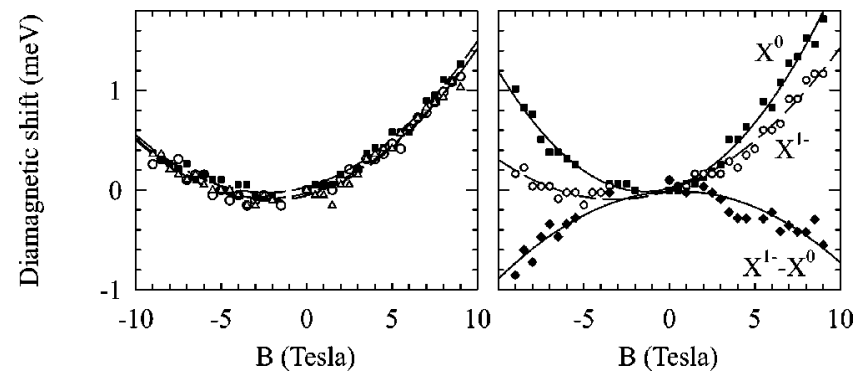

FIG. 2. Left: diamagnetic shift against magnetic field for the PL of a quantum dot emitting in the $1.31 \mathrm{eV}$ band. The three symbols correspond to $X^{0}, X^{1-}$, and $X^{2-}$ excitons. Right: diamagnetic shift for a quantum dot emitting in the $1.34 \mathrm{eV}$ band. The paramagnetic contribution due to charging is demonstrated by plotting the energy of $X^{1-}-X^{0}$. and we find that this is the case for all the investigated dots in the red band. In very clear contrast, the dots in the blue band have the remarkable property that the diamagnetic shift reduces with the addition of one electron. An example is shown in Fig. 2 where the neutral exciton has $\alpha$ $=16.6 \mu \mathrm{eV} / \mathrm{T}^{2}$, the singly charged exciton $8.7 \mu \mathrm{eV} / \mathrm{T}^{2}$. In other words, the extra electron makes a paramagnetic contribution of $\alpha=-7.9 \mu \mathrm{eV} / \mathrm{T}^{2}$ to the overall diamagnetism. We argue in the following that the paramagnetism is a consequence of Coulomb interactions in the QD.

In order to understand these experimental results, we present generic calculations in two different limits, strong and weak confinement. The aim is to make clear the role of few-body interactions in the diamagnetic shift, and thereby achieve a qualitative understanding of the experimental results. A complete quantitative agreement is probably only possible using the exact confinement potentials which are generally unknown for self-assembled QD's and it is not our purpose to explore this issue here. Importantly, we reach some wide-ranging conclusions which are independent of the form of the potential. For simplicity, we take a twodimensional (2D) parabolic potential for both electrons $(e)$ and holes $(h)$ of the type $V_{e(h)}=m_{e(h)} \Omega_{e(h)}^{2} r^{2} / 2$ where $\Omega_{e(h)}$ are the single particle frequencies, $m_{e(h)}$ the effective masses, and $r$ is the spatial in-plane coordinate. ${ }^{14}$ In the strong confinement regime, the single particle energies dominate such that the Coulomb energies can be treated as a firstorder perturbation to the single particle energies. ${ }^{15}$ The diamagnetic shift of the $n$-times negatively charged exciton $X^{n-}$ is proportional to $B^{2}$ in the limit where the electron (hole) cyclotron frequencies $\omega_{e(h)}^{\mathrm{cr}}=e B / m_{e(h)} \ll \Omega_{e(h)}$. The diamagnetic shift takes the form $\alpha=\alpha_{\mathrm{SP}}+\alpha_{\mathrm{C}}$ where SP (C) refers to the single-particle (Coulomb) contributions. We take $\alpha_{\mathrm{SP}}$ from the standard Fock-Darwin spectrum ${ }^{14}$ and we calculate $\alpha_{\mathrm{C}}$ for each charge state using an appropriate combination of Coulomb energy matrix elements. ${ }^{15}$ Results are plotted in Fig. 3 with parameters appropriate to InAs quantum dots showing how $\alpha$ depends on excess charge, with the changes becoming more important as the confinement weakens. The experimental diamagnetic shift is consistent with the calculations for an electron quantization energy of about $30 \mathrm{meV}$ which is a typical value for strongly confined InAs quantum dots. At this quantization energy, our calculations predict that the $\alpha$ 's for the $X^{0}, X^{1-}$, and $X^{2-}$ excitons differ by only $\sim 10 \%$ which is comparable to our experimental resolution in $\alpha$. The diamagnetic shifts of the red-band QD's are therefore consistent with the predictions of theory in the strong confinement limit.

In the other regime, weak confinement, the Coulomb energies dominate over the single particle energies. In the extreme case of a freely moving $X^{1-}$, the $X^{1-}$ has a negative magnetic dispersion, ${ }^{16,17}$ arising from the cyclotron motion: the electron mass in the final state is much less than the trion mass in the initial state. Experimentally, a weak paramagnetic dispersion for the $X^{1-}$ in a quantum well has been observed in fields of about $1 \mathrm{~T} \cdot{ }^{18,19}$ The same physics can be expected in the case of a charged exciton confined weakly to a quantum dot, and we prove this point by considering both 


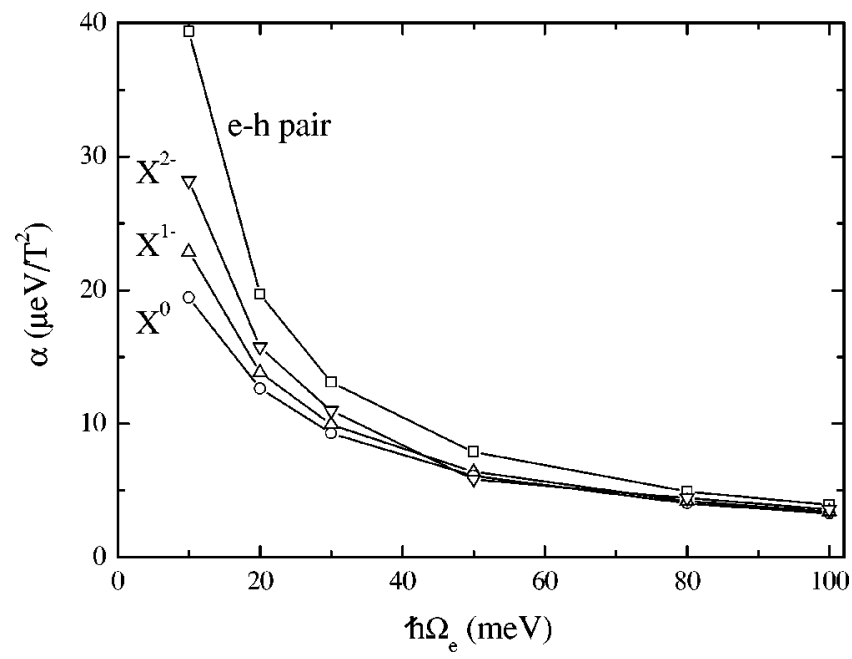

FIG. 3. Diamagnetic curvature $\alpha$ plotted against the electron quantization energy $\hbar \Omega_{e}$ for different excitonic charges. The curves are calculated in the strong confinement limit within first order perturbation theory. For $X^{2-}$ we show only the result for the emission into the triplet final state which is stronger than the emission into the singlet final state. It is assumed that the electron effective mass is 0.07 , the hole effective mass 0.25 and that the hole quantization energy is half that of the electron.

$X^{0}$ and $X^{1-}$ as pointlike particles in a parabolic confinement potential. In terms of lengths, this limit is appropriate when the dot size is much larger than the free exciton Bohr radius. In this limit, the effective potential is $2 V_{e}+V_{h}$ and so the center of mass motion of a trion is described by a harmonic wave function with radial and angular quantum numbers, both of which are 0 in the ground state. Using the single particle Fock-Darwin spectrum, we determine the $X^{1-}$ exciton energy to be $E^{1-}=E_{\mathrm{tr}}+\hbar\left[\Omega_{\mathrm{tr}}-\Omega_{e}(2 N+1)\right]$, where $E_{\mathrm{tr}}$ is the free $2 \mathrm{D}$ trion energy, $N$ is the radial quantum number of the electron left in the final state, and $\Omega_{\mathrm{tr}}^{2}=\left(2 m_{e} \Omega_{e}^{2}\right.$ $\left.+m_{h} \Omega_{h}^{2}\right) /\left(2 m_{e}+m_{h}\right)$. The interband selection rule on the envelope function dictates that the final state after photon emission should also have a zero angular momentum. Based on this, we find a $B^{2}$ dispersion of the PL emission energies with $\alpha_{N}^{1-}=\left(\hbar e^{2} / 8\right)\left[1 / m_{\mathrm{tr}}^{2} \Omega_{\mathrm{tr}}-(1+2 N) / m_{e}^{2} \Omega_{e}\right]$, which is negative for all $N$ because of the inequality $m_{\mathrm{tr}} \gg m_{e}$. The physical reason for the paramagnetism, i.e., $\alpha_{N}^{1-}<0$, is that the final state is more extended than the initial state. The dominant peak in the PL has $N=0$. The other peaks arise from shake-up processes in which the final state is an excited electron state. Such processes have already been investigated in the tunneling ${ }^{20}$ and PL spectroscopy of 2D systems, ${ }^{14,19,21,22}$ but to the best of our knowledge have not yet been observed in quantum dots systems. The intensity of the PL emission lines depends on an overlap integral of the trion and electron wave functions and this decreases rapidly with increasing $N$. Hence, theory in the weak confinement regime for $X^{1-}$ predicts a dominant PL peak with a paramagnetic behavior up to a small magnetic field.

Experimentally, the diamagnetic curvature of $X^{1-}$ in a blue-band QD is much smaller than that of $X^{0}$, but the overall dispersion is not paramagnetic. Also, we do not observe the shake-up peaks which are characteristic of weak confinement. Our explanation is that the blue-band QD's are in an intermediate regime. Interpolating between the two limits of our theory, in the intermediate regime the shake-up peaks will be weak and the diamagnetic contribution small and positive, giving us qualitative agreement with the experiment.

In conclusion, we report measurements of the diamagnetic shift of charged excitons in quantum dots in either the strong or the intermediate confinement regimes. In the strong confinement regime, the diamagnetic shift is small, and independent of charge. In the intermediate regime, the diamagnetic shift of a neutral exciton is larger, and there is a significant decrease with the addition of a single electron. The interpretation of our experiments is aided by theoretical models assuming either strong or weak confinement. For the weak confinement regime, we predict both an unusual paramagnetism and also the appearance of shake-up peaks in the emission. The novelty of these results is based on the fact that charged excitons are highly stable in quantum dots, which is not the case in bulk semiconductors and quantum wells.

We acknowledge S. Ulloa for fruitful discussions. This work was supported by the DFG (Grant No. SFB348) and EPSRC. One of us, A.O.G., acknowledges financial support from the Rufus Putnam Visiting Professorship and the Volkswagen Foundation.
${ }^{1}$ H. Drexler, D. Leonard, W. Hansen, J.P. Kotthaus, and P.M. Petroff, Phys. Rev. Lett. 73, 2252 (1994).

${ }^{2}$ S. Tarucha, D.G. Austing, T. Honda, R.J. van der Hage, and L.P. Kouwenhoven, Phys. Rev. Lett. 77, 3613 (1996).

${ }^{3}$ R.J. Warburton, C. Schäflein, D. Haft, F. Bickel, A. Lorke, K. Karrai, J.M. Garcia, W. Schoenfeld, and P.M. Petroff, Nature (London) 405, 926 (2000).

${ }^{4}$ A. Hartmann, Y. Ducommun, E. Kapon, U. Hohenester, and Elisa Molinari, Phys. Rev. Lett. 84, 5648 (2000).

${ }^{5}$ J.J. Finley, P.W. Fry, A.D. Ashmore, A. Lemaitre, A.I. Tartakovskii, R. Oulton, D.J. Mowbray, M.S. Skolnick, M. Hopkinson, P.D. Buckle, and P.A. Maksym, Phys. Rev. B 63, 161305 (2001).
${ }^{6}$ F. Findeis, M. Baier, A. Zrenner, M. Bichler, G. Abstreiter, U. Hohenester, and E. Molinari, Phys. Rev. B 63, 121309 (2001).

${ }^{7}$ W.G. van der Wiel, S. De Franceschi, T. Fujisawa, J.M. Elzerman, S. Tarucha, and L.P. Kouwenhoven, Science 289, 2105 (2000).

${ }^{8}$ R.P. Seisyan and B.P. Zakharchenya, in Landau Level Spectroscopy, edited by G. Landwehr and E.I. Rashba (Elsevier, Amsterdam, 1991), p. 347.

${ }^{9}$ J.-Y. Marzin, J.-M. Gérard, A. Izraël, and D. Barrier, Phys. Rev. Lett. 73, 716 (1994).

${ }^{10}$ J.M. Garcia, G. Medeiros-Ribeiro, K. Schmidt, T. Ngo, J.L. Feng, A. Lorke, and P.M. Petroff, Appl. Phys. Lett. 71, 2014 (1997). 
${ }^{11}$ G. Medeiros-Ribeiro, A.M. Bratkovski, T.I. Kamins, D.A.A. Ohlberg, and R.S. Williams, Science 279, 353 (1998).

${ }^{12}$ H. Lee, R. Lowe-Webb, T.J. Johnson, W. Yang, and P.C. Sercel, Appl. Phys. Lett. 73, 3556 (1998).

${ }^{13}$ R.J. Warburton, C. Schulhauser, D. Haft, C. Schäflein, K. Karrai, J.M. Garcia, W. Schoenfeld, and P.M. Petroff, Phys. Rev. B 65, 113303 (2002).

${ }^{14}$ L. Jacak, P. Hawrylak, and A. Wojs, Quantum Dots (Springer, Berlin, 1998).

${ }^{15}$ R.J. Warburton, B.T. Miller, C.S. Dürr, C. Bödefeld, K. Karrai, J.P. Kotthaus, G. Medeiros-Ribeiro, P.M. Petroff, and S. Huant, Phys. Rev. B 58, 16221 (1998).
${ }^{16}$ A.O. Govorov and A.V. Chaplik, Sov. Phys. JETP 72, 1037 (1991).

${ }^{17}$ B. Stébé, E. Feddi, and G. Munschy, Phys. Rev. B 35, 4331 (1987); A.V. Chaplik, Sov. Phys. JETP 92, 169 (2000).

${ }^{18}$ A.J. Shields, M. Pepper, M.Y. Simmons, and D.A. Ritchie, Phys. Rev. B 52, 7841 (1995).

${ }^{19}$ G. Finkelstein, H. Shtrikman, and I. Bar-Joseph, Phys. Rev. B 53, 12593 (1996).

${ }^{20}$ R.C. Ashoori, J.A. Lebens, N.P. Bigelow, and R.H. Silsbee, Phys. Rev. Lett. 64, 681 (1990).

${ }^{21}$ B.B. Goldberg, D. Heiman, A. Pinczuk, L. Pfeiffer, and K. West, Phys. Rev. Lett. 65, 641 (1990).

${ }^{22}$ A. Wojs and P. Hawrylak, Phys. Rev. B 55, 13066 (1997). 